\title{
Genotype distribution of hepatitis C virus in 952 cases from 2014 to 2016 in Hunan Province, China
}

Jian-Hua Lei, Xing Gong, Xin-Qiang Xiao, Zi Chen, Feng Peng

Department of Infectious Diseases, Second Xiangya Hospital, Central South University, Changsha, China

Submitted: 24 August 2017

Accepted: 17 September 2017

Arch Med Sci 2018; 14, 5: 1055-1060

DOI: https://doi.org/10.5114/aoms.2017.70664

Copyright $\odot 2017$ Termedia \& Banach

\section{Abstract}

Introduction: Few large-scale investigations on genotype (GT) distribution of hepatitis C virus (HCV) in Hunan Province, China, are reported.

Material and methods: We recruited all of the 952 patients in the census register of Hunan Province who were first diagnosed with HCV infection in the Second Xiangya Hospital, Central South University in 2014-2016. HCV genotypes were surveyed. The genotype distribution pattern was compared with those of the neighboring regions in China.

Results: Among the 952 patients, genotype 1 (GT1) (69.9\%) was the most common HCV genotype, followed by GT6 (19.0\%), GT3 (8.4\%), and GT2 (2.6\%). GT4 and GT5 were not found. One case had mixed infection of GT3 and GT6. Predominance of GT1 HCV was more evident in the patients aged $\geq 40$ years than in those aged $<40$ years $\left(79.5 \%\right.$ vs. $\left.47.9 \%, \chi^{2}=95.993, p<0.001\right)$. HCV genotype distribution had gender difference $\left(\chi^{2}=44.695, p<0.001\right)$, with GT3 and GT6 more prevalent in males than in females $\left(36.2 \%\right.$ vs. $18.2 \%, \chi^{2}=$ $39.088, p<0.001)$ while GT1 more prevalent in females than in males $(80.1 \%$ vs. $\left.60.3 \%, \chi^{2}=44.276, p<0.001\right)$. Though Hunan Province is located in central China, its HCV genotype priority was similar with the change trend in south and southwest China, while distinguished from those of other regions, in particular from the neighboring central province, Hubei Province.

Conclusions: HCV GT1 was the most predominant HCV genotype in Hunan Province, and GT6 and GT3 accounted for a significant percentage, especially in young patients. The HCV distribution pattern was more similar to those of the regions in south China.

Key words: epidemiology, hepatitis C virus, genotype.

\section{Introduction}

Hepatitis $C$ is a global pandemic disease. About 185 million people are infected or have previously been infected with hepatitis C virus (HCV), most of whom are progressing or have developed into the chronic stage. Moreover, new infections are emerging $[1,2]$. Hepatitis $C$ is moderately prevalent in East Asia including China, with an estimated prevalence rate of $0.29-3.2 \%$ in the general population [2-5]. Most HCV-infected patients are unaware that they are at higher risk for life-threatening diseases such as liver cirrhosis and hepatocellular carcinoma (HCC), whose incidences are predicted to rise in the coming decade [1, 2].

Epidemiological studies have confirmed that the prevalence of HCV has regional characteristics, and the HCV genotypes prevailing in different regions are widely divergent [5-7]. Knowing the distribution pattern

\author{
Corresponding author: \\ Prof. Jian-Hua Lei \\ Department of Infectious \\ Diseases \\ Second Xiangya Hospital \\ Central South University \\ 139 Middle \\ Renmin Road \\ 410011 Changsha \\ Hunan 410011, China \\ Phone: +86731 85292105 \\ E-mail: leijianhua73@163. \\ com
}


of virus genotypes is conducive to the control and prevention of the epidemic of HCV infection, and genotyping of the virus before anti-HCV treatment is essential for adjustment of the therapeutic regimen [4, 5, 8-10].

Prevailing patterns of HCV are evolving in China due to changes in transmission modes and other influencing factors such as immigration and global travel $[3,4,11,12]$. For example, HCV genotype 2 (HCV GT2), the second predominant HCV strain following HCV GT1 in north and central China, is prevalent in the southern and western provinces of China at a relatively lower level, while HCV GT6 is increasingly prevalent in Guangdong Province in south China, and HCV GT3 is thriving in Yunnan Province in southwest China [13, 14]. HCV GT6 has recently migrated from Guangdong Province to other provinces in the southern half of China [13].

Although patients from Hunan Province have been recruited in several large-size national-wide surveys on HCV infection in China, the number of the participants was limited, and no independent survey in Hunan Province was available [11, 12]. To our knowledge, there are few large reports on the distribution pattern of HCV genotypes in $\mathrm{Hu}$ nan Province in the authoritative literature about HCV infection and epidemiology. Here we documented the HCV genotype distribution pattern of 952 patients with a first-time confirmed diagnosis in Hunan Province in 2014-2016.

\section{Material and methods}

\section{Study participants}

We recruited 952 patients (494 males and 458 females) from the census register of Hunan Province who were confirmed for the first time to be infected with HCV by seropositive anti-HCV antibody and simultaneously detectable plasma HCV ribonucleic acid (RNA) in the Second Xiangya Hospital, Central South University in Hunan Province in 2014-2016. HCV was genotyped. In order to fully reveal the local HCV infection status, ethnicities and occupations of the patients, as well as their serum liver function indexes and complications such as liver cirrhosis, HCC and other diseases, were not considered in the exclusion criteria.

Collection of all the demographic data, clinical features and biological samples including the peripheral blood of the research objects fulfilled the requirements of medical ethics. The ethical review committees of the Second Xiangya Hospital of Central South University approved this study. Guidelines of the Declaration of Helsinki set by the committees were strictly followed. Due to the retrospective nature of the study, the written informed consent of the 611 participants enrolled in 2014 or 2015 was exempted. Written informed consent was obtained from the 341 participants enrolled in 2016.

\section{Diagnosis of HCV infection and genotyping of HCV}

Serum anti-HCV was assessed by an enzymelinked immunosorbent assay diagnostic kit (Zhuhai Livzon Diagnostics Inc., China) according to the manufacturer's instructions. Quantitation of plasma HCV RNA was assessed in a 7500 real-time polymerase chain reaction (PCR) system (Applied Biosystems Inc., USA) using a HCV RNA quantitative fluorescence diagnostic kit (Sansure biotech Inc. Ltd, Chi$\mathrm{na}$ ), and the lower detection limit was $25 \mathrm{IU} / \mathrm{ml}$.

A multiplex one-step real-time fluorescent RTPCR assay based on the analysis of the conserved sequence of the HCV genome was carried out in an SLAN-96P real-time fluorescence quantitative PCR instrument (Hongshi Medical Technology Co., Ltd, Shanghai, China) using a hepatitis $C$ virus genotype diagnostic kit (PCR-fluorescence probing) (Sansure biotech Inc. Ltd, Hunan, China). This protocol could discern HCV genotypes 1-6 and determine subtype $1 b[15,16]$. It could detect mixed $\mathrm{HCV}$ infections of multiple genotypes on the basis of fluorescent signals.

\section{Statistical analysis}

All statistical analyses were performed with IBM SPSS Statistics version 20.0, using descriptive statistical indexes such as median, the $25^{\text {th }}$ or the $75^{\text {th }}$ centile, etc. The one-sample Kolmogorov-Smirnov test was used to verify the normal distribution of data sets. The $\chi^{2}$ test and Kruskal-Wallis $\mathrm{H}$ test were performed for comparison of ratios or medians. The Levene test was used to verify the homogeneity of variance. For all these tests, a $p$-value less than 0.05 was considered statistically significant.

\section{Results}

\section{Distribution of HCV genotypes and subtypes}

HCV genotypic diversity was observed (Table I). Genotype distribution patterns from 2014 to 2016 are relatively stable (Pearson $\chi^{2}=10.038, p=$ 0.262). GT1 was the most common HCV genotype across Hunan Province, comprising $69.9 \%$ of the overall first-time confirmed HCV infected patients, followed by GT6 (19.0\%) and GT3 (8.4\%). GT2 was rare $(2.6 \%)$. GT4 and GT5 were not found. One patient had mixed infections of genotypes 3 and 6 .

\section{Agewise distribution of the HCV infected patients with different virus genotypes}

The age of the 952 patients recruited took an abnormal distribution (Kolmogorov-Smirnov 
Table I. Distribution of HCV genotypes in 952 HCV cases in Hunan Province, China, 2014-2016

\begin{tabular}{|ccccccc|}
\hline Year & $\boldsymbol{N}$ & \multicolumn{5}{c|}{ HCV genotype, $\boldsymbol{n}(\%)$} \\
\cline { 3 - 7 } & & $\mathbf{1}$ & $\mathbf{2}$ & $\mathbf{3}$ & $\mathbf{6}$ & $\mathbf{3}+\mathbf{6}$ \\
\hline 2014 & 200 & $133(66.5)$ & $9(4.5)$ & $20(10.0)$ & $37(18.5)$ & $1(0.5)$ \\
\hline 2015 & 411 & $286(69.6)$ & $7(1.7)$ & $34(8.3)$ & $84(20.4)$ & $0(0.0)$ \\
\hline 2016 & 341 & $246(72.1)$ & $9(2.6)$ & $26(7.6)$ & $60(17.6)$ & $0(0.0)$ \\
\hline Total & 952 & $665(69.9)$ & $25(2.6)$ & $80(8.4)$ & $181(19.0)$ & $1(0.1)$ \\
\hline
\end{tabular}

Pearson $\chi^{2}=10.038, p=0.262$.

test, $Z=1.586, p=0.013)$, with the peak at age 37-53 years (Figure 1, Table II). Patients infected with HCV of different genotypes had specific age distribution features. Seventy-five percent of the HCV GT1 infected patients were older than 42 and the median was 49 years old, while GT3 and GT6 were more prevalent in younger patients (IQR, interquartile range from the $25^{\text {th }}$ to the $75^{\text {th }}$ centile, 33-45 and 33-43 years old, respectively). HCV GT2 was detected in fewer cases, manifesting a sporadic distribution (Figure 1, Table II). The participants were grouped into 3-39 and 40-79 years age groups. For the patients aged 40 years and above, the predominance of HCV GT1 was more evident than the patients younger than 40 years old $\left(79.5 \%\right.$ vs. $47.9 \%, \chi^{2}=95.993, p<$ $0.001)$. For the patients younger than 40 years old, although HCV GT1 was still the main prevalent isolate (140 cases, $47.9 \%$ ), the constitutions of GT3 and GT6 obviously increased and their sum was approximate to that of GT1 (145 cases, 49.7\%) (Figure 2).

\section{Comparison of the distribution of the HCV genotypes on gender basis}

The male and female patients had a similar HCV genotype distribution pattern (Table III). However, the proportions of various HCV genotypes were significantly different between males and females $\left(\chi^{2}=44.695, p<0.001\right)$. The proportion of GT1 was higher in females $\left(\chi^{2}=44.276, p<\right.$ $0.001)$, while the proportions of GT3 and GT6 were obviously higher in males $\left(\chi^{2}=39.088, p<0.001\right)$.

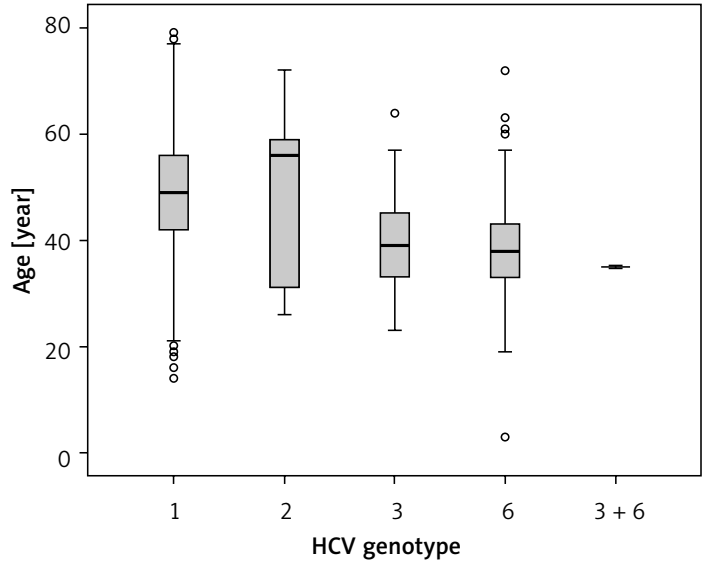

Figure 1. Age span of HCV infected patients with various HCV genotypes in Hunan Province, China, 2014-2016

$\chi^{2}=144.329, p<0.001$ (heterogeneity of variance, Kruskal-Wallis $\mathrm{H}$ test).

\section{Comparison of the HCV genotype distribution patterns of Hunan Province and the neighboring regions in China}

HCV GT1 was, without exception, the most predominant HCV strain all through China, and GT4 and GT5 were not found. Proportions of GT2, GT3 and GT6 were distinctly different among different regions of China. GT2 was the second predominant HCV strain in northern, central and eastern China, but only a few sporadic cases were found in Hunan Province. Instead, GT6 and GT3 were more common. The distribution of HCV genotypes in Hunan Province was more

Table II. Age of HCV infected patients with different HCV genotypes in Hunan Province, China, 2014-2016

\begin{tabular}{|lccccc|}
\hline HCV genotype & $\boldsymbol{N}$ & \multicolumn{5}{c|}{ Age [years] } \\
\cline { 2 - 6 } & & Median & IQR & Min. & Max. \\
\hline 1 & 665 & 49 & $42-56$ & 14 & 79 \\
\hline 2 & 25 & 56 & $31-59$ & 26 & 72 \\
\hline 3 & 80 & 39 & $33-45$ & 23 & 64 \\
\hline 6 & 181 & 38 & $33-43$ & 3 & 72 \\
\hline $3+6$ & 1 & - & - & 35 & 35 \\
\hline Total & 952 & 47 & $37-53$ & 3 & 79 \\
\hline
\end{tabular}

$\chi^{2}=144.329, p<0.001$ (heterogeneity of variance, Kruskal-Wallis $\mathrm{H}$ test). IQR - interquartile range from $25^{\text {th }}$ to $75^{\text {th }}$ centile. 


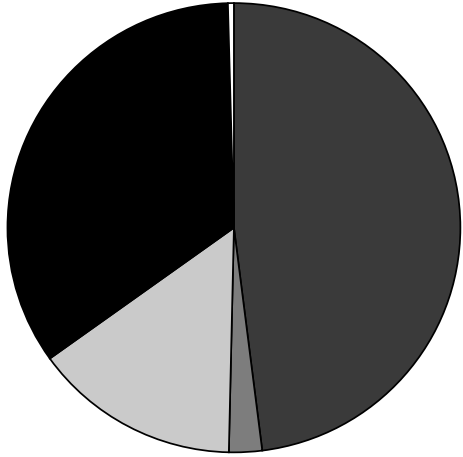

Age: 3-39 years

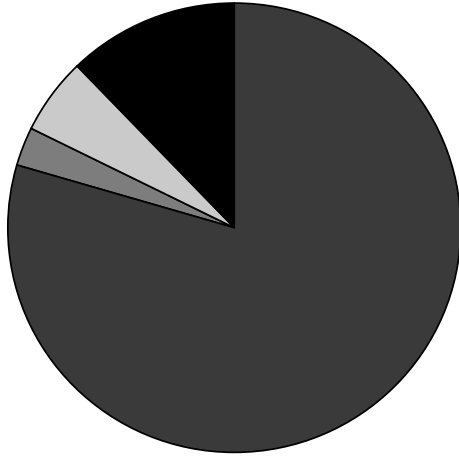

Age: $40-79$ years

HCV genotype: $\square 1 \quad \square 2 \quad \square 3 \quad \square 6 \quad \square 3+6$

Figure 2. Agewise HCV genotype distribution of HCV infected patients in Hunan Province, China, in 2014-2016

$\chi^{2}=105.069, p<0.001$

similar to the change trend in south and southwest China (Table IV). What is particularly noteworthy is the distinct difference between Hunan and Hubei Province, two neighboring provinces in central China.

\section{Discussion}

Although there is still no effective vaccine for HCV infection prevention, based on the epidemiological regularity of this communicable disease in the population, the Chinese medical personnel have made great achievements in HCV infection control by means of screening of serum anti-HCV in blood donors, strict management of blood transfusion and legitimate clinical utilization of blood products [3-5]. Analysis of HCV genotype distribution is conducive to the prevention and antiviral treatment of HCV infection [4, 5, 8, 9].

Hunan Province is a populous province located in central China, with $68,220,000$ inhabitants. Its economic development level cannot ensure

Table III. HCV genotype distribution on gender basis in Hunan Province, China, 2014-2016

\begin{tabular}{|lcccccc|}
\hline Gender & \multicolumn{3}{c}{ HCV genotype, $n$ (\%) } & \multicolumn{2}{c|}{ Total, $n$ (\%) } \\
\cline { 2 - 6 } & 1 & 2 & 3 & 6 & $3+6$ \\
\hline Male & $298(60.3)$ & $17(3.4)$ & $55(11.1)$ & $123(24.9)$ & $1(0.2)$ & $494(100)$ \\
\hline Female & $367(80.1)$ & $8(1.7)$ & $25(5.5)$ & $58(12.7)$ & $0(0.0)$ & $458(100)$ \\
\hline Total & $665(69.9)$ & $25(2.6)$ & $80(8.4)$ & $181(19.0)$ & $1(0.1)$ & $952(100)$ \\
\hline
\end{tabular}

Overall: $\chi^{2}=44.695, p<0.001 ;$ GT1/non-GT1: $\chi^{2}=44.276, p<0.001 ;$ GT3 + GT6/GT1 + GT2: $\chi^{2}=39.088, p<0.001$.

Table IV. Distribution of HCV genotypes in different regions of China

\begin{tabular}{|c|c|c|c|c|c|c|c|c|c|}
\hline \multirow[t]{2}{*}{ Regions } & \multicolumn{3}{|c|}{ Cases } & \multicolumn{5}{|c|}{ HCV genotypes (\%) } & \multirow{2}{*}{$\begin{array}{l}\text { Publication } \\
\text { time }\end{array}$} \\
\hline & Year & Source & $N$ & 1 & 2 & 3 & 6 & Multi & \\
\hline China & 2011 & Outpatients & 997 & 59.2 & 25.4 & 9.0 & 6.4 & 2.1 & 2014 [11] \\
\hline North China & & & 181 & 58.6 & 16.0 & 6.1 & 5.7 & 2.1 & \\
\hline East China & & & 222 & 73.3 & 7.8 & 5.3 & 2.9 & 1.0 & \\
\hline Central China & & & 243 & 66.8 & 15.3 & 1.6 & 2.9 & 2.5 & \\
\hline South China & & & 155 & 41.4 & 8.6 & 23.7 & 20.4 & 2.6 & \\
\hline West China & & & 211 & 45.7 & 26.7 & 11.6 & 7.0 & 0.9 & \\
\hline Hunan & 2014-2016 & Outpatients & 952 & 69.9 & 2.6 & 8.4 & 19.0 & 0.1 & Our data \\
\hline Guangdong & 2008-2011 & $\begin{array}{l}\text { Volunteer } \\
\text { blood donors }\end{array}$ & 258 & 44.2 & 2.7 & 13.6 & 39.5 & 0 & 2014 [13] \\
\hline Hubei & $2013-2014$ & Outpatients & 177 & 83.0 & 13.0 & 2.9 & 1.1 & 0 & 2015 [17] \\
\hline Southwest China* & 2004-2011 & Outpatients & 1137 & 35.8 & 11.1 & 33.7 & 19.4 & 0 & $2012[14]$ \\
\hline
\end{tabular}

${ }^{*}$ Source of the cases [14]: Chongqing Municipality 61.7\%, Sichuan Province 21.2\%, Guizhou Province 13.9\%, other southwest provinces (Yunnan, Guangxi and Xizang) $3.2 \%$. 
screening of HCV infection in all residents, just as screening for prostate cancer by the prostate-specific antigen test in the elderly Polish population is also impractical [17]. More and more cases are being or have been confirmedly diagnosed as HCV infected patients in recent years. However, there are no large-scale reports on the distribution pattern of HCV genotypes in Hunan Province in the authoritative literature $[11,12]$. Our data of the HCV genotype distribution pattern in 952 patients with a confirmed diagnosis for the first time in recent years in Hunan Province has filled a big gap.

Similar to other districts of China $[3,4,7,11-$ 14,18 ], HCV GT1 was the most predominant HCV strain in Hunan Province, and GT4 and GT5 were not found. HCV GT2, which is reported to be the second predominant HCV strain in northern and central China [3, 4, 7, 11, 12], was only detected in $2.6 \%$ of the 952 cases in Hunan Province, while GT6 ranked as the second most prevalent genotype and GT3 the third. Its HCV genotype distribution pattern was similar to the change trend in south and southwest China. It has been reported that HCV GT6 has rapidly spread from Guangdong Province to other regions and a pattern with increase in GT3 was found in southern and western China $[13,14,19,20]$. This observation seemed to be in line with the situation in Hunan, a central province of China. A likely explanation may be the population migration between Hunan Province and other places, especially Guangdong Province, together with transmission route changes since the economic reform in China decades ago [21].

Many diseases, including infectious diseases and metabolic diseases, have gender or age specific distribution differences [22]. Agewise distribution of HCV genotypes of the 952 cases indicated that for patients older than 40 years, the genotypes were more concentrated. Otherwise, the HCV genotypes were obviously dispersed among patients younger than 40 years. It may be associated with more diverse transmission routes in patients aged below 40 years. Patients with HCV infection resulting from intravenous drug use (IDU) [23] or high-risk sexual behavior are relatively young, and the infection has a relatively short incubation period $[19,20$, 24]. HCV genotype distribution showed an overall difference between genders, and the proportions of GT3 and GT6 were higher in males than in females. The IDU and high-risk sexual behavior constitute the main transmission routes of $\mathrm{HCV}$ among young male people in southern and western China. It is conjectured that the situation is similar in Hunan Province, consistent with the nationwide spread of GT3 and GT6 in recent years $[3,4,7,13,14,19,20]$. These facts suggest that advice and education on a healthy lifestyle should be strengthened to prevent high-risk behaviors such as IDU and unsafe sexual behavior in young men.

Transmission routes in some patients, especially most of those infected with HCV GT1 in Chinese rural areas, could be traced back to blood transfusion or blood donation 20-30 years before [3, 14, $19,21,25,26]$. These patients had quite a long clinical incubation period. Once chronic HCV infection progresses to the clinical stage, it poses great harm to patients and society. Nowadays, many rapid and inexpensive detection methods of HCV infection have been generalized, and great achievements have been made in the treatment of HCV infection [4, 5], so it is necessary to screen $\mathrm{HCV}$ infection among the population who had blood transfusion or donation in the 1980s-1990s $[21,25,26]$.

The study delineated the HCV genotype distribution in a large sample and over a long duration in Hunan Province for the first time. The agewise and genderwise distribution of the HCV genotypes indicated the changing tendency of the HCV infection, and could provide evidence for further study of the local HCV epidemic and formulating targeted prevention and control strategy. However, the study had the following limitations. The sample size was not large enough, and the sample was not quite representative, for the participants were not randomly sampled from the patients diagnosed with hepatitis $C$ in all the medical institutions in Hunan Province. The study would benefit from further analysis of the HCV genetic data associated with the different transmission routes of the virus such as phylogenetic study.

In conclusion, HCV GT1 was the most predominant HCV genotype in Hunan Province, China, and GT6 and GT3 accounted for a significant percentage, especially in young patients. Though Hunan Province is located in central China, its HCV genotype distribution was similar to the change trend in south and southwest China, but was distinct from those of other regions, in particular from the neighboring central province, Hubei Province. It is necessary to screen HCV infection among the population with blood transfusion or donation in the 1980s-1990s and to propagate a healthy lifestyle in young men.

\section{Acknowledgments}

Institutional review board statement: The study was reviewed and approved by the Review Board. The number of the approval was 2015-S032.

Informed consent statement: Due to the retrospective nature of the study, written informed consent of the 611 participants enrolled in 2014 or 2015 was exempted. Written informed consent 
was obtained from the 341 participants enrolled in 2016.

\section{Conflict of interest}

The authors declare no conflict of interest.

\section{References}

1. Thomas DL. Global control of hepatitis C: where challenge meets opportunity. Nat Med 2013; 19: 850-8.

2. Mohd Hanafiah K, Groeger J, Flaxman AD, Wiersma ST. Global epidemiology of hepatitis $C$ virus infection: new estimates of age-specific antibody to HCV seroprevalence. Hepatology 2013; 57: 1333-42.

3. Cui Y, Jia J. Update on epidemiology of hepatitis B and C in China. J Gastroenterol Hepatol 2013; 28 (Suppl 1): 7-10.

4. Chinese Society of Hepatology, Chinese Society of Infectious Diseases. The guideline of prevention and treatment for hepatitis C: a 2015 update. Zhonghua Gan Zang Bing Za Zhi 2015; 23: 906-23.

5. Omata M, Kanda T, Wei L, et al. APASL consensus statements and recommendations for hepatitis $C$ prevention, epidemiology, and laboratory testing. Hepatol Int 2016; 10: 681-701.

6. Gower E, Estes C, Blach S, Razavi-Shearer K, Razavi H. Global epidemiology and genotype distribution of the hepatitis C virus infection. J Hepatol 2014; 61 (1 Suppl): S45-57.

7. Zhang Y, Chen LM, He M. Hepatitis C virus in mainland China with an emphasis on genotype and subtype distribution. Virol J 2017; 14: 41

8. Omata M, Kanda T, Wei L, et al. APASL consensus statements and recommendation on treatment of hepatitis C. Hepatol Int 2016; 10: 702-26.

9. AASLD/IDSA HCV Guidance Panel. Hepatitis C guidance: AASLD-IDSA recommendations for testing, managing, and treating adults infected with hepatitis C virus Hepatology 2015; 62: 932-54.

10. European Association for the Study of the Liver. EASL Recommendations on Treatment of Hepatitis C 2016. J Hepatol 2017; 66: 153-94.

11. Rao H, Wei L, Lopez-Talavera JC, et al. Distribution and clinical correlates of viral and host genotypes in Chinese patients with chronic hepatitis C virus infection. J Gastroenterol Hepatol 2014; 29: 545-53.

12. Lu L, Wang M, Xia W, et al. Migration patterns of hepatitis $C$ virus in China characterized for five major subtypes based on samples from 411 volunteer blood donors from 17 provinces and municipalities. J Virol 2014; 88: 7120-9.

13. Rong $\mathrm{X}, \mathrm{Xu} \mathrm{R}$, Xiong $\mathrm{H}$, et al. Increased prevalence of hepatitis $C$ virus subtype $6 a$ in China: a comparison between 2004-2007 and 2008-2011. Arch Virol 2014; 159: 3231-7.

14. Yan Z, Fan K, Wang Y, Fan Y, Tan Z, Deng G. Changing pattern of clinical epidemiology on hepatitis $C$ virus infection in Southwest China. Hepat Mon 2012; 12: 196-204.

15. Nakatani SM, Santos CA, Riediger IN, et al. Development of hepatitis $C$ virus genotyping by real-time PCR based on the NS5B region. PLoS One 2010; 5: e10150.

16. Rolfe KJ, Wreghitt TG, Alexander GJ, Curran MD. A realtime Taqman method for hepatitis $C$ virus genotyping and methods for further subtyping of isolates. Methods Mol Biol 2009; 510: 55-71.
17. Prajsner A, Chudek J, Szybalska A, Piotrowicz K, Zejda J, Więcek A; PolSenior Study Group. Socioeconomic determinants of prostate-specific antigen testing and estimation of the prevalence of undiagnosed prostate cancer in an elderly Polish population based on the PolSenior study. Arch Med Sci 2016; 12: 1028-35.

18. Peng J, Lu Y, Liu W, et al. Genotype distribution and molecular epidemiology of hepatitis C virus in Hubei, Central China. PLoS One 2015; 10: e0137059.

19. Ju W, Yang S, Feng S, et al. Hepatitis C virus genotype and subtype distribution in Chinese chronic hepatitis C patients: nationwide spread of HCV genotypes 3 and 6. Virol J 2015; 12: 109.

20. Zhang Z, Yao Y, Wu W, et al. Hepatitis C virus genotype diversity among intravenous drug users in Yunnan Province, Southwestern China. PLoS One 2013; 8: e82598.

21. Yin W, Huang C, Qiu F, et al. Risk factors of hepatitis C virus transmission and genotype distribution in former blood donors from Chinese rural area. BMC Public Health 2015; 15: 184

22. Pajak A, Szafraniec K, Polak M, et al. Prevalence of familial hypercholesterolemia: a meta-analysis of six large, observational, population-based studies in Poland. Arch Med Sci 2016; 12: 687-96.

23. Salemovic D, Pesic-Pavlovic I, Jevtovic D, et al. Intravenous drug use - an independent predictor for HCV genotypes 3 and 4 infection among HIV/HCV co-infected patients. Arch Med Sci 2017; 13: 652-8.

24. Jiao Y, Zhang X, Wang C, et al. Hepatitis C virus subtype and evolution characteristic among drug users, men who have sex with men, and the general population in Beijing, China. Medicine (Baltimore) 2016; 95: e2688.

25. Lu L, Tong W, Gu L, et al. The current hepatitis C virus prevalence in China may have resulted mainly from an officially encouraged plasma campaign in the 1990s: a coalescence inference with genetic sequences. J Virol 2013; 87: 12041-50.

26. Wang CB, Cheng ZX, Chen JJ, et al. Epidemiological characteristics and risk factors of hepatitis $C$ virus genotype 1 infection: a national epidemiological survey of Chinese Han population. Eur Rev Med Pharmacol Sci 2016; 20: 1052-6. 